\title{
Kaon-baryon coupling constants in the QCD sum rule approach
}

\author{
Seungho Choe* \\ Department of Physics, Hiroshima University, Higashi-Hiroshima 739-8526, Japan
}

\begin{abstract}
We improve our previous QCD sum rule calculation on $g_{K N \Lambda}$ and $g_{K N \Sigma}$ coupling constants by including the contributions from higher dimensional condensates, $\left\langle\bar{q} g_{s} \sigma \cdot G q\right\rangle$ and $\langle\bar{q} q\rangle\left\langle\frac{\alpha_{s}}{\pi} G^{2}\right\rangle$, in the OPE. It is found that the contribution of these condensates is non-negligible compared to that of the quark condensates. Using a best-fit analysis we find $\left|g_{K N \Lambda}\right|=2.49 \pm 1.25$ and $\left|g_{K N \Sigma}\right|=0.395 \pm 0.377$.
\end{abstract}

PACS numbers: $13.75 \mathrm{Jz}, 11.55 \mathrm{Hx}$

*E-mail : schoe@hirohe.hepl.hiroshima-u.ac.jp 


\section{INTRODUCTION}

To understand kaon-nuclear physics, it is important to know the hadronic coupling strengths involving the kaons. Among them, $g_{K N \Lambda}$ and $g_{K N \Sigma}$ are the most relevant coupling constants. In contrast to $g_{\pi N N}$, however, the determination of these kaon couplings has some difficulties both in the experimental side and in the theoretical side, e.g. see [1].

Among other theoretical approaches, QCD sum rule method [2 4] has been used to extract these kaon couplings. However, compared to the large number of works devoted to $g_{\pi N N}$, there have been only few QCD sum rule estimates on $g_{K N \Lambda}$ and $g_{K N \Sigma}$ [5 9], for which there are still ambiguities in among the calculations. Thus the results are quite different from each other. More detailed analyses are needed both experimentally and theoretically to understand this discrepancy, and to understand kaon-nuclear physics.

In Ref. [5.7], the OPE was calculated only up to the leading term coming from the quark condensate and to leading order in $m_{s}$ in the sum rule structure proportional to $\not i \gamma_{5}$. However, the next leading term, dimension $5\left\langle\bar{q} g_{s} \sigma \cdot G q\right\rangle$ may contribute to the OPE side with considerable amount as in nucleon mass sum rule [10]. In addition, operators of dimension 7 may also be important in the OPE side as a further power correction. Thus, in this paper we re-analyze our QCD sum rule calculation including higher dimensional condensates, such as $\left\langle\bar{q} g_{s} \sigma \cdot G q\right\rangle$ and $\langle\bar{q} q\rangle\left\langle\frac{\alpha_{s}}{\pi} G^{2}\right\rangle$, and study the contribution of these condensates on the previous results.

In Sec. II we present our sum rules for $g_{K N \Lambda}$ and $g_{K N \Sigma}$, and Sec. III we discuss some uncertainties in our sum rules and summarize our results.

\section{QCD SUM RULES FOR $g_{K N \Lambda}$ AND $g_{K N \Sigma}$}

We will closely follow the procedures given in Refs. [11, 3, 5, [7]. Consider the three point function constructed of the two baryon currents $\eta_{B}, \eta_{B^{\prime}}$ and the pseudoscalar meson current $j_{5}$.

$$
A\left(p, p^{\prime}, q\right)=\int d x d y\left\langle 0\left|T\left(\eta_{B^{\prime}}(x) j_{5}(y) \bar{\eta}_{B}(0)\right)\right| 0\right\rangle e^{i\left(p^{\prime} \cdot x-q \cdot y\right)} .
$$

In order to obtain $g_{K N \Lambda}$, we will use the following currents for the nucleon and the $\Lambda$ [12,3].

$$
\begin{gathered}
\eta_{N}=\epsilon_{a b c}\left(u_{a}^{T} C \gamma_{\mu} u_{b}\right) \gamma_{5} \gamma^{\mu} d_{c} \\
\eta_{\Lambda}=\sqrt{\frac{2}{3}} \epsilon_{a b c}\left[\left(u_{a}^{T} C \gamma_{\mu} s_{b}\right) \gamma_{5} \gamma^{\mu} d_{c}-\left(d_{a}^{T} C \gamma_{\mu} s_{b}\right) \gamma_{5} \gamma^{\mu} u_{c}\right]
\end{gathered}
$$

where $\mathrm{u}$ and $\mathrm{d}$ are the up and down quark fields ( $a, b$ and $c$ are color indices), $T$ denotes the transpose in Dirac space, and $C$ is the charge conjugation matrix. For the $K^{-}$we choose the current

$$
j_{K^{-}}=\bar{s} i \gamma_{5} u \text {. }
$$

The general expression for $A\left(p, p^{\prime}, q\right)$ has the following form 


$$
\begin{aligned}
A\left(p, p^{\prime}, q\right) & =F_{1}\left(p^{2}, p^{\prime 2}, q^{2}\right) i \gamma_{5}+F_{2}\left(p^{2}, p^{2}, q^{2}\right) q i \gamma_{5} \\
& +F_{3}\left(p^{2}, p^{\prime 2}, q^{2}\right) p i \gamma_{5}+F_{4}\left(p^{2}, p^{\prime 2}, q^{2}\right) \sigma^{\mu \nu} \gamma_{5} q_{\mu} p_{\nu}^{\prime},
\end{aligned}
$$

where $q=p^{\prime}-p$ and $P=\frac{p+p^{\prime}}{2}$. Recently, in Ref. 13] it was reported that in the case of $g_{\pi N N}$ the $\sigma^{\mu \nu} \gamma_{5}$ structure is independent of the effective models employed in the phenomenological side and further provides the $\pi N N$ coupling with less uncertainties from QCD parameters. Motivated by this result $g_{K N \Lambda}$ and $g_{K N \Sigma}$ were calculated from this structure in Refs. [8,9]. In this paper, however, we construct the sum rule for only the $q i \gamma_{5}$ structure as before, and compare this with our previous one.

On the phenomenological side, keeping the first two terms we have

$$
\begin{aligned}
& \lambda_{N} \lambda_{\Lambda} \frac{M_{B}}{\left(p^{2}-M_{N}^{2}\right)\left(p^{2}-M_{\Lambda}^{2}\right)}\left(\phi i \gamma_{5}\right) g_{K N \Lambda} \frac{1}{q^{2}-m_{K}^{2}} \frac{f_{K} m_{K}^{2}}{2 m_{q}} \\
+ & \lambda_{N} \lambda_{\Lambda^{*}} \frac{M_{B}^{\prime}}{\left(p^{2}-M_{N}^{2}\right)\left(p^{2}-M_{\Lambda^{*}}^{2}\right)}\left(\phi i \gamma_{5}\right) g_{K N \Lambda^{*}} \frac{1}{q^{2}-m_{K}^{2}} \frac{f_{K} m_{K}^{2}}{2 m_{q}} \\
+ & \text { higher resonances, }
\end{aligned}
$$

where $M_{B}=\frac{1}{2}\left(M_{N}+M_{\Lambda}\right)$, and $M_{B}^{\prime}=\frac{1}{2}\left(M_{N}-M_{\Lambda}^{*}\right)$. Here $\Lambda^{*}$ means the $\Lambda(1405)$, and we introduce (-) sign for the $\Lambda$ (1405) mass because it is a negative parity state. However, this is not relevant in the following calculation. $\lambda_{N}, \lambda_{\Lambda}$ and $\lambda_{\Lambda^{*}}$ are the coupling strengths of the baryons to their currents. $m_{q}$ is the average of the quark masses, $f_{K}$ the kaon decay constant and $m_{K}$ the kaon mass. We take $f_{K}=0.160 \mathrm{GeV}$ and $m_{s}=0.150 \mathrm{GeV}$.

As for the OPE side, the new contribution from the quark-gluon condensates is given by

$$
-\sqrt{\frac{2}{3}} \frac{7}{2^{4} 3 \pi^{2}} \ln \left(-p^{2}\right)\left(\left\langle\bar{q} g_{s} \sigma \cdot G q\right\rangle+\left\langle\bar{s} g_{s} \sigma \cdot G s\right\rangle\right),
$$

and from dimension 7 ops.

$$
+\sqrt{\frac{2}{3}} \frac{5}{2^{3} 3^{2}} \frac{1}{p^{2}}(\langle\bar{q} q\rangle+\langle\bar{s} s\rangle)\left\langle\frac{\alpha_{s}}{\pi} G^{2}\right\rangle,
$$

where we take the limit $p^{2} \rightarrow p^{2}$ and let $\langle\bar{u} u\rangle=\langle\bar{d} d\rangle \equiv\langle\bar{q} q\rangle,\left\langle\bar{u} g_{s} \sigma \cdot G u\right\rangle=\left\langle\bar{d} g_{s} \sigma \cdot\right.$ $G d\rangle \equiv\left\langle\bar{q} g_{s} \sigma \cdot G q\right\rangle$. Here we collect only the terms which contribute to the $\not / q^{2}$ structure such as Figs. 11 and 2. Using the standard values for $\left\langle\bar{s} g_{s} \sigma \cdot G s\right\rangle=0.8\left\langle\bar{q} g_{s} \sigma \cdot G q\right\rangle$ and $\left\langle\bar{q} g_{s} \sigma \cdot G q\right\rangle=m_{0}^{2}\langle\bar{q} q\rangle=0.8\langle\bar{q} q\rangle$ [14] the sum rule after Borel transformation to $p^{2}=p^{2}$ becomes

$$
\begin{aligned}
& \lambda_{N} \lambda_{\Lambda} \frac{M_{B}}{M_{\Lambda}^{2}-M_{N}^{2}}\left(e^{-M_{N}^{2} / M^{2}}-e^{-M_{\Lambda}^{2} / M^{2}}\right) g_{K N \Lambda} \frac{f_{K} m_{K}^{2}}{2 m_{q}}+A\left(e^{-M_{N}^{2} / M^{2}}-e^{-M_{\Lambda^{*}}^{2} / M^{2}}\right)= \\
&-\sqrt{\frac{2}{3}}\left(\frac{33}{40 \pi^{2}} E_{1} M^{4}+\left(\frac{11 m_{s}^{2}}{60 \pi^{2}}-\frac{21}{100 \pi^{2}}\right) E_{0} M^{2}+\left(\frac{m_{s}}{3}\langle\bar{s} s\rangle+\frac{1}{8}\left\langle\frac{\alpha_{s}}{\pi} G^{2}\right\rangle\right)\right)\langle\bar{q} q\rangle .
\end{aligned}
$$

Here, A is the unknown constant coming from $\lambda_{\Lambda^{*}} \cdot g_{K N \Lambda^{*}}$, and

$$
E_{i}=1-\sum_{k=0}^{i} \frac{s_{0}^{k}}{k !\left(M^{2}\right)^{k}} e^{-\frac{s_{0}}{M^{2}}}
$$


where $s_{0}$ is a continuum threshold. One should be cautious, however, that there may be non-accounted terms, which can not be neglected by using this simple Borel transformation [15, 16.

For $\lambda_{N}$ and $\lambda_{\Lambda}$, we use the values obtained from the following baryon sum rules for the $N$ and $\Lambda$ [12,3]:

$$
\begin{gathered}
E_{2}^{N} M^{6}+b E_{0}^{N} M^{2}+\frac{4}{3} a^{2}=2(2 \pi)^{4} \lambda_{N}^{2} e^{-M_{N}^{2} / M^{2}} \\
E_{2}^{\Lambda} M^{6}+\frac{2}{3} a m_{s}(1-3 \gamma) E_{0}^{\Lambda} M^{2}+b E_{0}^{\Lambda} M^{2}+\frac{4}{9} a^{2}(3+4 \gamma)=2(2 \pi)^{4} \lambda_{\Lambda}^{2} e^{-M_{\Lambda}^{2} / M^{2}},
\end{gathered}
$$

where $a \equiv-(2 \pi)^{2}\langle\bar{q} q\rangle, b \equiv \pi^{2}\left\langle\frac{\alpha_{s}}{\pi} G^{2}\right\rangle$, and $\gamma \equiv\langle\bar{s} s\rangle /\langle\bar{q} q\rangle-1 \simeq-0.2$. We use different thresholds for $\lambda_{N}$ and $\lambda_{\Lambda}$ in Eqs. (11) and (12). We take $s_{N}=(1.440 \mathrm{GeV})^{2}$ for the nucleon sum rule and $s_{\Lambda}=(1.405 \mathrm{GeV})^{2}$ for the $\Lambda$ sum rule considering the next excited nucleon and $\Lambda$ state, respectively.

$g_{K N \Lambda}$, however, does not display a plateau as a function of the Borel mass. This is because there is no usual power correction term like $\left(\frac{a}{M^{2}}, \frac{b}{M^{4}}\right.$, and so on $)$ in the r.h.s. of Eq. (9) even including up to dimension 7 operators. We need more higher dimensional operators to get those terms. Thus, in this case we prefer to use a best-fit method. Eq. (9) has the following form :

$$
g_{K N \Lambda} \cdot f_{1}\left(M^{2}\right)+A \cdot f_{2}\left(M^{2}\right)=f_{3}\left(M^{2}\right)
$$

Then, we get $g_{K N \Lambda}$ and the unknown constant A by minimizing $\left(g_{K N \Lambda} \cdot f_{1}+A \cdot f_{2}-f_{3}\right)^{2}$ with a fixed $s_{0}$ and an appropriate Borel interval:

$$
\int_{M_{\min }^{2}}^{M_{\max }^{2}}\left(g_{K N \Lambda} \cdot f_{1}+A \cdot f_{2}-f_{3}\right)^{2} d M^{2}=\text { minimum }
$$

We fix the continuum threshold $s_{0}=2.074 \mathrm{GeV}^{2}$ taking into account the next term from the $\mathrm{N}(1440)$, i.e., $N(1440) \rightarrow \Lambda$, in the phenomenological side.

The Borel interval $M^{2}$ is restricted by the following conditions : OPE convergence and pole dominance. The lower limit of $M^{2}, M_{\min }^{2}$ is determined as the value at which the contribution of the highest dimensional operators is less than $10 \%$ of total OPE. The upper limit $M_{\text {max }}^{2}$ is determined by restricting the continuum contribution to be less than $50 \%$. Then, we get

$$
\begin{aligned}
\left|g_{K N \Lambda}\right| & =2.49 \\
|A| & =0.00174 \mathrm{GeV}^{7},
\end{aligned}
$$

and the Borel interval $(0.478,1.068) \mathrm{GeV}^{2}$ for basic inputs (i.e., $\langle\bar{q} q\rangle=-(0.230 \mathrm{GeV})^{3}$, $\left\langle\frac{\alpha_{s}}{\pi} G^{2}\right\rangle=0.012 \mathrm{GeV}^{4}, m_{s}=0.150 \mathrm{GeV}$, and $\left.m_{0}^{2}=0.8 \mathrm{GeV}^{2}\right)$. Here we denote the absolute value because we can not determine signs of the coupling strengths $\left(\lambda_{N}\right.$, $\lambda_{\Lambda}$ and $\lambda_{\Lambda^{*}}$ ) in the baryon sum rules. We also calculate the average deviation $\bar{\delta} \equiv$ $\sum_{i}^{N}\left|1-R H S\left(M_{i}^{2}\right) / L H S\left(M_{i}^{2}\right)\right| / N=8.8 \times 10^{-2}$ to test the reliability of our fitting, and it shows that the deviation is less than $10 \%$. 
Table 1 shows variations of $g_{K N \Lambda}$ for other input parameters, which are coming from the uncertainty of the basic inputs. For example, the first line in Table 1 shows that $\left|g_{K N \Lambda}\right|=$ 3.11 (or 1.93) if we change the quark condensate to $\langle\bar{q} q\rangle=-(0.210 \mathrm{GeV})^{3}$ (or $\left.-(0.250 \mathrm{GeV})^{3}\right)$ while other basic inputs are fixed. In the last line we take $m_{0}^{2}=0.6 \mathrm{GeV}^{2}$ from the lowest value of the standard QCD sum rule estimate [14], and $1.4 \mathrm{GeV}^{2}$ which was evaluated in the instanton vacuum in Ref. [17]. Total variation is about \pm 1.25 on the above $g_{K N \Lambda}$ value. On the other hand, the unknown constant $|A|$ varies from 0.00120 to $0.00203 \mathrm{GeV}^{7}$.

Next, consider $g_{K N \Sigma}$. The current of $\Sigma^{\circ}$ is obtained by making an $\mathrm{SU}(3)$ rotation from the nucleon current [18]

$$
\eta_{\Sigma}=\sqrt{2} \epsilon_{a b c}\left[\left(u_{a}^{T} C \gamma_{\mu} s_{b}\right) \gamma_{5} \gamma^{\mu} d_{c}+\left(d_{a}^{T} C \gamma_{\mu} s_{b}\right) \gamma_{5} \gamma^{\mu} u_{c}\right]
$$

In this case the contribution of the quark-gluon condensate is given by

$$
-\sqrt{2} \frac{1}{2^{4} 3 \pi^{2}} \ln \left(-p^{2}\right)\left(\left\langle\bar{q} g_{s} \sigma \cdot G q\right\rangle+\left\langle\bar{s} g_{s} \sigma \cdot G s\right\rangle\right)
$$

and from dimension 7 ops.

$$
+\sqrt{2} \frac{1}{2^{3} 3^{2}} \frac{1}{p^{2}}(\langle\bar{q} q\rangle+\langle\bar{s} s\rangle)\left\langle\frac{\alpha_{s}}{\pi} G^{2}\right\rangle .
$$

Then, within the same approximation as before we get the following sum rule.

$$
\begin{array}{r}
\lambda_{N} \lambda_{\Sigma} \frac{M_{B}}{M_{\Sigma}^{2}-M_{N}^{2}}\left(e^{-M_{N}^{2} / M^{2}}-e^{-M_{\Sigma}^{2} / M^{2}}\right) g_{K N \Sigma} \frac{f_{K} m_{K}^{2}}{2 m_{q}}+B\left(e^{-M_{N^{*}}^{2} / M^{2}}-e^{-M_{\Sigma}^{2} / M^{2}}\right)= \\
+\sqrt{2}\left(\frac{3}{40 \pi^{2}} E_{1} M^{4}+\left(\frac{m_{s}^{2}}{60 \pi^{2}}+\frac{3}{100 \pi^{2}}\right) E_{0} M^{2}-\frac{1}{40}\left\langle\frac{\alpha_{s}}{\pi} G^{2}\right\rangle\right)\langle\bar{q} q\rangle,
\end{array}
$$

where $M_{B}=\frac{1}{2}\left(M_{N}+M_{\Sigma}\right)$ and $N^{*}$ is $\mathrm{N}(1440)$. B is the unknown constant coming from $\lambda_{N^{*}} \cdot g_{K N^{*} \Sigma}$. Again for $\lambda_{\Sigma}$, we take the value from the following sum rule for the $\Sigma$ [12, 3]:

$$
E_{2}^{\Sigma} M^{6}-2 a m_{s}(1+\gamma) E_{0}^{\Sigma} M^{2}+b E_{0}^{\Sigma} M^{2}+\frac{4}{3} a^{2}=2(2 \pi)^{4} \lambda_{\Sigma}^{2} e^{-M_{\Sigma}^{2} / M^{2}} .
$$

We fix the continuum threshold $s_{\Sigma}=(1.660 \mathrm{GeV})^{2}$ considering the next $\Sigma$ state, $\Sigma(1660)$.

Using the continuum threshold $s_{0}=2.356 \mathrm{GeV}^{2}$ taking into account the next term from the $\mathrm{N}(1535)$, i.e., $N(1535) \rightarrow \Sigma$, in the phenomenological side we get

$$
\begin{aligned}
\left|g_{K N \Sigma}\right| & =0.395 \\
|B| & =0.00148 \mathrm{GeV}^{7}
\end{aligned}
$$

for the same basic inputs. The Borel interval is $(0.488,1.584) \mathrm{GeV}^{2}$ and the average deviation of the fit $\bar{\delta}$ is $9.7 \%$ in this case. We present the variation of $g_{K N \Sigma}$ on other parameters in Table 【1. The total variation is about \pm 0.377 . On the other hand, $|B|$ varies from 0.00117 to $0.00184 \mathrm{GeV}^{7}$. 


\section{DISCUSSION}

SU(3) symmetry, using de Swart's convention [19], predicts

$$
\begin{aligned}
& g_{K N \Lambda}=-\frac{1}{\sqrt{3}}\left(3-2 \alpha_{D}\right) g_{\pi N N} \\
& g_{K N \Sigma}=+\left(2 \alpha_{D}-1\right) g_{\pi N N}
\end{aligned}
$$

where $\alpha_{D}$ is the fraction of the $\mathrm{D}$ type coupling, $\alpha_{D}=\frac{D}{D+F}$. In Table III we compare our results with previous QCD sum rule estimates [6,86,9] and an $\mathrm{SU}(3)$ symmetry prediction, where we denote the error-bar allowing for SU(3) symmetry breaking at the $20 \%$ level. Here we take $\alpha_{D}$ from a recent analysis of hyperon semi-leptonic decay data by Ratcliffe, $\alpha_{D}=0.64$ [20], and $g_{\pi N N}$ from an analysis of the $n p$ data by Ericson et al. [21], $g_{\pi N N}=13.43$. A comparison to fitting analyses of experimental data [22] is also provided. SU(3) symmetry predicts $\left|g_{K N \Lambda} / g_{K N \Sigma}\right|=3.55$ taking $\alpha_{D}=0.64$, while our results show that this ratio is 6.30 using the basic inputs, and the order of SU(3) symmetry breaking is rather huge.

Let us remark on $g_{\pi N N}$ which was calculated in Ref. [11,3] using the three-point function method. After including dimension 5 and 7 condensates as in the previous section the sum rule becomes

$$
\begin{array}{r}
\lambda_{N}^{2} \frac{e^{-M_{N}^{2} / M^{2}}}{M^{2}} M_{N} g_{\pi N N} \frac{f_{\pi} m_{\pi}^{2}}{\sqrt{2} m_{q}}+C\left(e^{-M_{N^{*}}^{2} / M^{2}}-e^{-M_{N}^{2} / M^{2}}\right)= \\
-\left(\frac{1}{\pi^{2}} E_{1} M^{4}-\frac{1}{5 \pi^{2}} E_{0} M^{2}+\frac{1}{9}\left\langle\frac{\alpha_{s}}{\pi} G^{2}\right\rangle\right)\langle\bar{q} q\rangle,
\end{array}
$$

where $\mathrm{C}$ is the unknown constant from $\lambda_{N^{*}} \cdot g_{\pi N N^{*}}$ and $f_{\pi}=0.133 \mathrm{GeV}$. The contribution of the quark-gluon condensates in the OPE side is important as in the $g_{K N \Lambda}$ and $g_{K N \Sigma}$ sum rules. In this case we use the PCAC relation $f_{\pi}^{2} m_{\pi}^{2}=-4 m_{q}\langle\bar{q} q\rangle$ first, then the quark condensate becomes an overall factor on both sides. However, the coupling strength $\lambda_{N}$ is still related to the quark condensate as shown in Eq. (11).

Following the same method in the previous section, and using $\langle\bar{q} q\rangle=-(0.230 \mathrm{GeV})^{3}$, $\left\langle\frac{\alpha_{s}}{\pi} G^{2}\right\rangle=0.012 \mathrm{GeV}^{4}$, and $s_{0}=2.074 \mathrm{GeV}^{2}$ as a pure continuum threshold we get

$$
\begin{aligned}
\left|g_{\pi N N}\right| & =3.65 \pm 2.31 \\
|C| & =0.00261 \pm 0.00091 \mathrm{GeV}^{7}
\end{aligned}
$$

the Borel interval $(0.460,1.110) \mathrm{GeV}^{2}$, and the average deviation of the fit $\bar{\delta} 9.3 \%$ at the central value. Here the uncertainty comes from using different input parameters, i.e. $\langle\bar{q} q\rangle=$ $-(0.210 \mathrm{GeV})^{3}\left(\right.$ or $\left.-(0.250 \mathrm{GeV})^{3}\right),\left\langle\frac{\alpha_{s}}{\pi} G^{2}\right\rangle=0.015 \mathrm{GeV}^{4}$, and $m_{0}^{2}=0.6$ (or $\left.1.4 \mathrm{GeV}^{2}\right)$ as before. In this case the most error bar comes from uncertainties of the quark condensate, i.e. from the coupling strength $\lambda_{N}$.

Now, let us discuss some uncertainties in our sum rules. In Eqs. (91), (19) and (23) the contribution of the quark-gluon condensate is about $25 \%, 40 \%$, and $20 \%$, respectively, of the leading term at $M^{2}=1 \mathrm{GeV}^{2}$. Thus the accurate value of this condensate is one of important factors in our sum rules, and a more precise estimate may be needed (e.g., see Ref. [23]). 
As we mentioned before, we need more higher dimensional operators to get some power correction terms in our sum rules. Their contribution will be much smaller than that of dimension 7 operators at the relevant Borel region around $M^{2} \sim 1 \mathrm{GeV}^{2}$. However, those operators may contribute because the lower limit of the Borel interval for each coupling constant is much less than $1 \mathrm{GeV}^{2}$ in our sum rules.

We find that the coupling constants become 2 or 3 times larger than the previous ones if we take the coupling strengths $\left(\lambda_{N}, \lambda_{\Lambda}\right.$ and $\left.\lambda_{\Sigma}\right)$ from the chiral-odd baryon sum rules [12, 3]. For example, we get 7.04, 0.890, and 14.49 for $\left|g_{K N \Lambda}\right|,\left|g_{K N \Sigma}\right|$, and $\left|g_{\pi N N}\right|$, respectively, for the basic inputs. Because the coupling strengths from each baryon sum rule (the chiral-even and chiral-odd) are not the same in the whole Borel region and the discrepancy between the coupling strengths is larger in the low Borel region, we get quite different coupling constants. Of course, it should be judged by the stability of the sum rule whether one chooses the coupling strengths from the chiral-even sum rules or those from the chiral-odd sum rules.

As a final remark, in the case of $g_{\pi N N}$ it was shown that there is a higher pseudoscalar resonance contamination from the $\pi(1300)$ and $\pi$ (1800) in the three-point function method [24]. Maybe there is a similar contamination from the $\mathrm{K}(1460)$ and $\mathrm{K}(1830)$ [25] on the kaonbaryon couplings. Although the masses of the $\mathrm{K}(1460)$ and $\mathrm{K}(1830)$ are quite uncertain and these states need further experimental confirmation, we can briefly estimate the contribution of the $\mathrm{K}(1460)$ as done in Ref. [24]. Using the parameters from recent works [26], we get

$$
\left[\frac{f_{M} m_{M}^{2}}{Q^{2}+m_{M}^{2}}\right]_{Q^{2}=1 \mathrm{GeV}^{2}}=21.3 \text { and } 2.2 \mathrm{MeV}
$$

for the kaon and $\mathrm{K}(1460)$, respectively. Here $f_{M}$ is the decay constant and $m_{M}$ is the meson mass. We take $f_{K}=108 \mathrm{MeV}, f_{K(1460)}=3.3 \mathrm{MeV}$ and $m_{K}=496 \mathrm{MeV}, m_{K(1460)}=1.45$ $\mathrm{GeV}$ in Ref. [26]. Comparing the values in Eq. (25) to those for the pion and $\pi$ (1300) [26], i.e. 1.7 and $0.4 \mathrm{MeV}$, the contamination from the excited kaon state on the kaon-baryon couplings seems smaller than that from the excited pion state on $g_{\pi N N}$.

In summary, including higher dimensional condensates we re-analyze our previous QCD sum rule estimate on $g_{K N \Lambda}$ and $g_{K N \Sigma}$ in the $\not i \gamma_{5}$ structure. The contribution of dimension 5 quark-gluon condensates is comparable to that of the leading term, and the present result is much different from the previous one.

\section{ACKNOWLEDGMENTS}

The author thanks Prof. Su H. Lee for valuable discussions and comments. This work is supported by Research Fellowship of the Japan Society for the Promotion of Science (JSPS). 


\section{TABLES}

TABLE I. $g_{K N \Lambda}$ and its variations. Other inputs mean other possible inputs coming from the uncertainty of the basic inputs.

\begin{tabular}{ccc}
\hline \hline basic inputs & other inputs & variations \\
\hline$\langle\bar{q} q\rangle=-(0.230 \mathrm{GeV})^{3}$ & $-(0.210 \mathrm{GeV})^{3},-(0.250 \mathrm{GeV})^{3}$ & $+0.62,-0.53$ \\
$\left\langle\frac{\alpha_{s}}{\pi} G^{2}\right\rangle=0.012 \mathrm{GeV}^{4}$ & $0.015 \mathrm{GeV}^{4}$ & +0.45 \\
$m_{s}=0.150 \mathrm{GeV}$ & $0.120,0.180 \mathrm{GeV}$ & $-0.36,+0.30$ \\
$m_{0}^{2}=0.8 \mathrm{GeV}^{2}$ & $0.6,1.4 \mathrm{GeV}^{2}$ & $-0.19,0.02$ \\
\hline \hline
\end{tabular}

TABLE II. $g_{K N \Sigma}$ and its variations. The same as in Table $\mathbb{E}$.

\begin{tabular}{ccc}
\hline \hline basic inputs & other inputs & variations \\
\hline$\langle\bar{q} q\rangle=-(0.230 \mathrm{GeV})^{3}$ & $-(0.210 \mathrm{GeV})^{3},-(0.250 \mathrm{GeV})^{3}$ & $+0.057,-0.061$ \\
$\left\langle\frac{\alpha_{s}}{\pi} G^{2}\right\rangle=0.012 \mathrm{GeV}^{4}$ & $0.015 \mathrm{GeV}^{4}$ & +0.201 \\
$m_{s}=0.150 \mathrm{GeV}$ & $0.120,0.180 \mathrm{GeV}$ & $-0.084,+0.086$ \\
$m_{0}^{2}=0.8 \mathrm{GeV}^{2}$ & $0.6,1.4 \mathrm{GeV}^{2}$ & $+0.067,-0.198$ \\
\hline \hline
\end{tabular}

TABLE III. Comparison of coupling constants.

\begin{tabular}{ccc}
\hline \hline Sources & $g_{K N \Lambda}$ & $g_{K N \Sigma}$ \\
\hline SU(3) with 20\% breaking & $-16.0 \sim-10.7$ & $3.0 \sim 4.5$ \\
Experimental fitting [22] & -13.7 & 3.9 \\
Ref. [6] & $10 \pm 6$ & $3.6 \pm 2$ \\
Ref. [8] & $2.37 \pm 0.09$ & $0.025 \pm 0.015$ \\
Ref. [9] & $10 \pm 2$ & $0.75 \pm 0.15$ \\
Present work & $2.49 \pm 1.25$ & $0.395 \pm 0.377$ \\
\hline \hline
\end{tabular}




\section{REFERENCES}

[1] M.K. Cheoun, B.S. Han, B.G. Yu, and Il-Tong Cheon, Phys. Rev. C54, 1811 (1996).

[2] M.A. Shifman, A.I. Vainshtein, and V.I. Zakharov, Nucl. Phys. B147, 385, 448 (1979).

[3] L.J. Reinders, H. Rubinstein, and S. Yazaki, Phys. Rep. 127, 1 (1985).

[4] S. Narison, "QCD Spectral Sum Rules", World Scientific Lecture Notes in Physics, Vol. 26 (1989); and references therein.

[5] S. Choe, M.K. Cheoun, and Su H. Lee, Phys. Rev. C53, 1363 (1996).

[6] B. Krippa, Phys. Lett. B420, 13 (1998).

[7] S. Choe, Phys. Rev. C57, 2061 (1998).

[8] M.E. Bracco, F.S. Navarra, and M. Nielsen, Phys. Lett. B454, 346 (1999).

[9] T.M. Aliev and M. Savc1, Phys. Rev. C61, 045201 (2000).

[10] D.B. Leinweber, Ann. Phys. 254, 328 (1997).

[11] L.J. Reinders, H. Rubinstein, and S. Yazaki, Nucl. Phys. B213, 109 (1983).

[12] B.L. Ioffe, Nucl. Phys. B188, 317 (1981); B191, 591(E) (1981).

[13] H. Kim, Su H. Lee, and M. Oka, Phys. Lett. B453, 199 (1999); Phys. Rev. D60, 034007 (1999); H. Kim, T. Doi, M. Oka, and Su H. Lee, nucl-th/0002011.

[14] V.M. Belyaev and B.L. Ioffe, Sov. Phys. JETP 56, 493 (1982); 57, 761 (1983).

[15] B.L. Ioffe, Phys. At. Nucl. 58, 1408 (1995).

[16] H. Kim, to appear in Prog. Theor. Phys. , nucl-th/9906081.

[17] M.V. Polyakov and C. Weiss, Phys. Lett. B387, 841 (1996).

[18] C.B. Chiu, J. Pasupathy, and S.L. Wilson, Phys. Rev. D32, 1786 (1985).

[19] J.J. de Swart, Rev. Mod. Phys. 35, 916 (1963).

[20] P.G. Ratcliffe, Phys. Lett. B365, 383 (1996).

[21] T.E.O. Ericson, B. Loiseau, J. Blomgren, and N. Olsson, $\pi$ N News Letter 12, 6 (1996).

[22] R.G.E. Timmermans, T.A. Rijken, and J.J. de Swart, Nucl. Phys. A585, 143c (1995).

[23] A.A. Ovchinnikov and A.A. Pivovarov, Yad. Fiz. 48, 1135 (1988).

[24] K. Maltman, Phys. Rev. C57, 69 (1998).

[25] Particle Data Group, Eur. Phys. J. C3, 1 (1998).

[26] M.K. Volkov and V.L. Yudichev, and D. Ebert, J. Phys. G25, 2025 (1999); M.K. Volkov and V.L. Yudichev, hep-ph/9906371. 


\section{FIGURES}

FIG. 1. Contribution of dimension 5 operators. The solid lines are quark propagators and the wavy line is a gluon propagator. The dotted line denotes a meson.

FIG. 2. Contribution of dimension 7 operators. The same as in Fig. 1] 

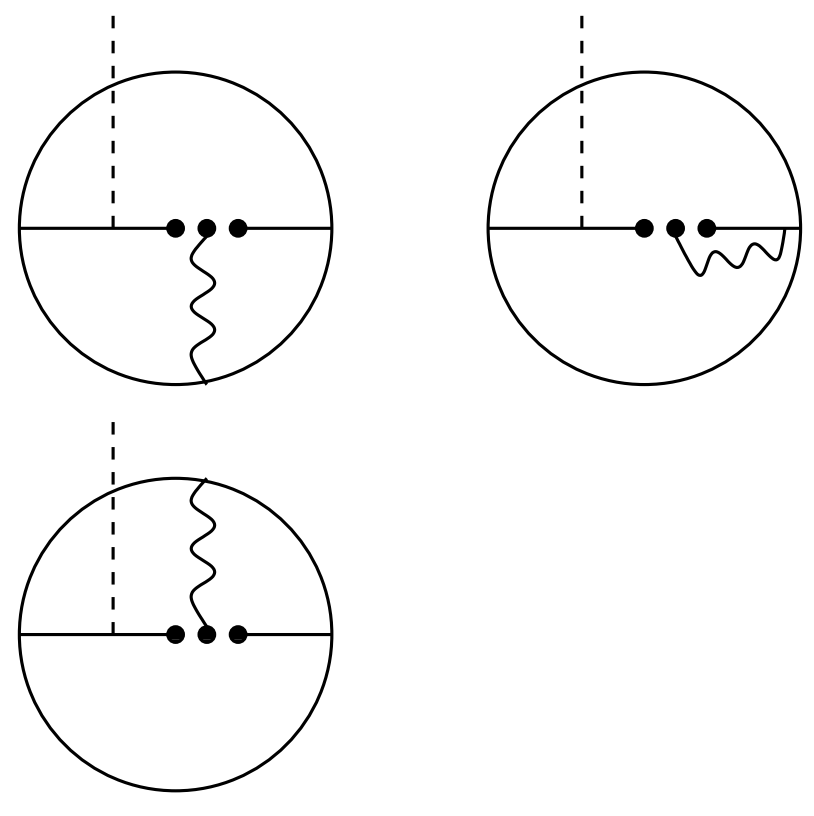

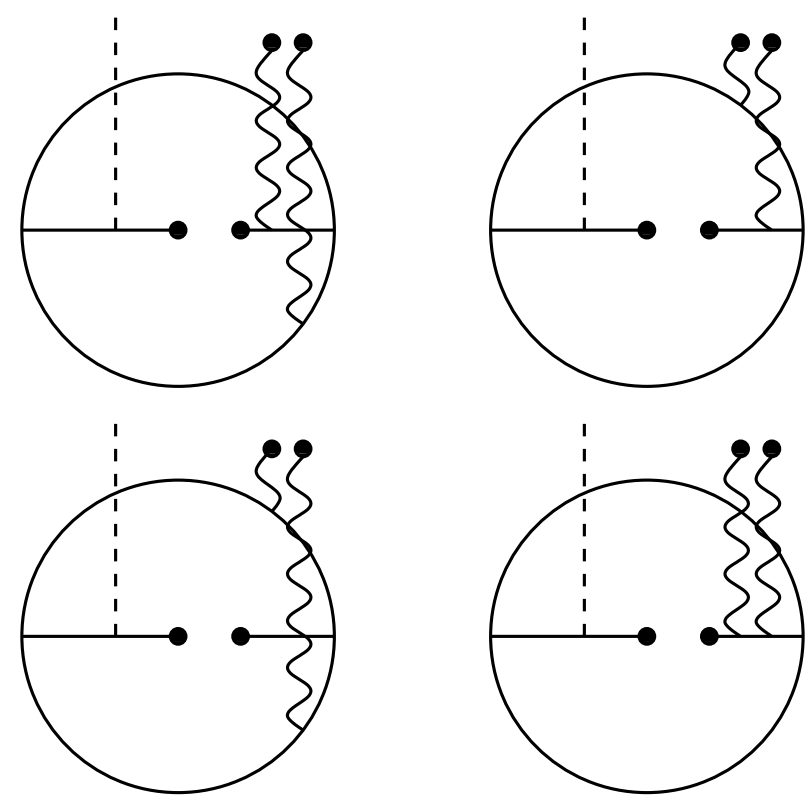